\title{
Abnormalities in Platelet Behaviour in Acute Illnesses
}

\author{
J. R. HAMPTON,* M.A., B.M., M.R.C.P. ; J. R. A. MITCHELL,* M.A., M.D., D.PHIL., M.R.C.P.
}

Brit. med. F., 1966, 1, 1078-1080

Abnormalities in platelet behaviour have been described in a variety of pathological conditions. Increased platelet-to-glass adhesiveness has been found after surgical operations (Payling Wright, 1942), in ischaemic heart disease (McDonald and Edgill, 1957), pulmonary embolus (Hirsh and McBride, 1965), disseminated sclerosis (Field and Caspary, 1964), and homocystinuria (McDonald et al., 1964), while Emmons and Mitchell (1965) showed that aggregating agents caused increased plateletto-platelet adhesion in the post-operative period.

We have shown (Hampton and Mitchell, 1966a) that agents which cause platelet aggregation induce changes in platelet surface charge as estimated by measurement of the platelet electrophoretic mobility. Low concentrations of these agents cause an increase in mobility, and concentrations of the order which cause aggregation in a stirred system cause a decrease in mobility. During these investigations we found that platelets from patients with a wide variety of acute illnesses behaved abnormally, and these abnormalities are described in this paper.

\section{Methods}

Platelet-rich and platelet-poor citrated plasma were prepared, and the platelets were brought into contact with glass as described previously (Hampton and Mitchell, 1966b). Plateletrich citrated plasma was diluted 1 in 10 with either plateletpoor citrated plasma or some other medium as described below, and the post-contact electrophoretic mobility of platelets was measured in the horizontal capillary apparatus developed by Bangham et al. (1958). All measurements were made at $25^{\circ} \mathrm{C}$. with a potential gradient of $2.66 \mathrm{~V} / \mathrm{cm}$. The mobilities are expressed as $\mu / \mathrm{sec} . / \mathrm{V} / \mathrm{cm}$.

After measuring the post-contact mobility of the platelets adenosine diphosphate (A.D.P.) or noradrenaline (both from Sigma), dissolved in $0.85 \%$ (w/v) saline, were added to the platelet-rich/platelet-poor citrated plasma mixtures, and the platelet mobility was measured after 10 minutes' incubation at $25^{\circ} \mathrm{C}$. At least three concentrations of each agent were tested : one which caused the maximum increase in mobility, one 10 times more dilute, which caused a small increase, and one 10 times more concentrated, which caused a significant decrease in mobility. These three estimations enabled a "dose-response" curve for changes in platelet mobility to be constructed.

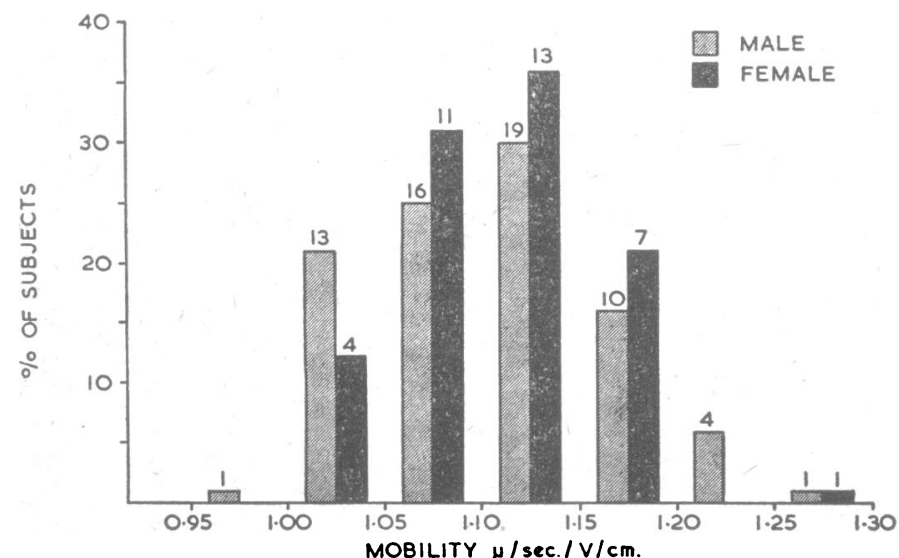

FIG. 1.-Freyuency distribuuon of pos. contact platelet electrophoretic mobility

\section{Post-contact Electrophoretic Mobility}

The distribution of post-contact mobilities was the same in healthy and ill subjects, and Fig. 1 shows the frequencydistribution histogram for 100 individuals $(9$ healthy subjects; 13 patients with psychiatric conditions, 11 with ischaemic heart disease, 11 with peripheral vascular disease and cerebrovascular accidents, and 9 with peptic ulcer; the remaining 47 patients had been admitted with haematological conditions, with acute and chronic inflammatory conditions such as pneumonia and collagen disease, and for acute and elective surgery of benign and malignant conditions).

\section{Changes in Mobility Produced by Aggregating Agents}

\section{Effects of Myocardial Infarction}

Seven patients (six men aged 45-64 and one woman aged 56) suffering from acute myocardial infarction were studied. It was found that their platelets were more sensitive to A.D.P. and noradrenaline than those of normal subjects in that a very low concentration of these agents induced the maximum increase in mobility. In six of the seven patients the maximum increase was produced by $0.0005 \mu \mathrm{g}$. of A.D.P. or noradrenaline per $\mathrm{ml}$. in the final mixture, which is one-hundredth the concentration required in a normal subject; in the remaining case the maximum increase was induced by $0.005 \mu \mathrm{g} . / \mathrm{ml}$. As with normal subjects, there was a biphasic response to A.D.P. or noradrenaline, a tenfold increase in concentration above that which gave the maximum increase in mobility producing a decrease in mobility.

The change in sensitivity was probably the result of the infarction, because in one patient blood was obtained within one hour of the onset of pain and the dose-response curve was found to be normal, the maximum mobility being induced by $0.05 \mu \mathrm{g}$. of A.D.P. or noradrenaline per ml. Next day, however, the maximum increase in mobility was obtained with one-tenth this concentration- $0.005 \mu \mathrm{g} . / \mathrm{ml}$. In two further patients blood was obtained six hours and eight hours after the onset of pain, and, although the response to A.D.P. and noradrenaline was already abnormal, the platelets were less sensitive than they were the following day. In the remaining patients blood was obtained 12 to 24 hours after the onset of pain, and in these patients the platelets were found to be at their most sensitive.

The increased platelet sensitivity to A.D.P. and noradrenaline became progressively less marked and ultimately disappeared (Fig. 2 shows dose-response curves for A.D.P. in one patient on three days in the week following infarction). Four patients were studied at intervals after the infarction until the platelets had returned to their original sensitivity. The dose-response curves for A.D.P. and noradrenaline were changed in parallel, the maximum increase in mobility being obtained with the same concentration of either agent. In a fifth patient there was a parallel increase in sensitivity to the two agents during the first week. By the eighth day the sensitivity to noradrenaline had returned to normal, the maximum increase in mobility being induced by $0.05 \mu \mathrm{g} . / \mathrm{ml}$., but with A.D.P. the maximum

\footnotetext{
* Department of the Regius Professor of Medicine, Radcliffe Infirmary,
} Oxford. 
increase was induced by $0.005 \mu \mathrm{g} . / \mathrm{ml}$. This patient was studied for three months, and there was no further change: we shall present evidence separately that dissociation between the sensitivity of the platelets to A.D.P. and noradrenaline is characteristic of certain chronic, as opposed to acute, diseases. Apart from this single instance, where the platelets remained abnormally sensitive to A.D.P., all the patients had returned to their pre-infarction state within nine days, and it seemed that those with less severe lesions returned to normal more rapidly than the others.

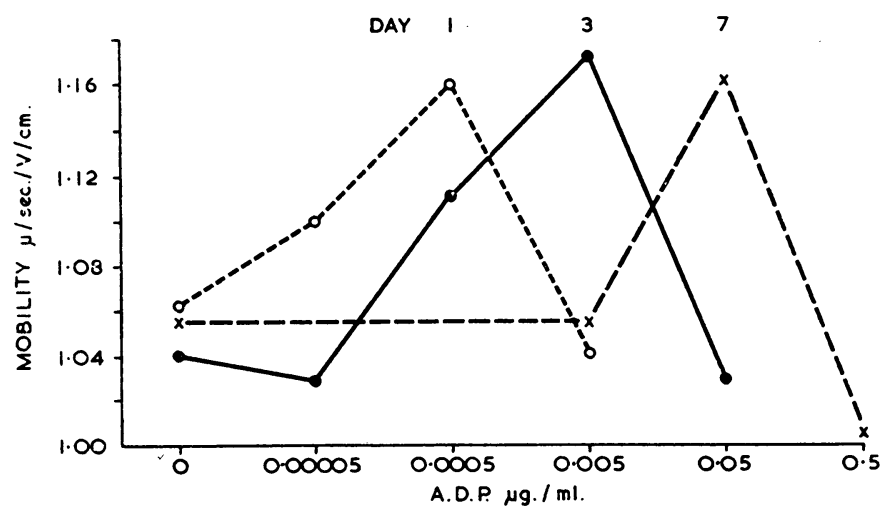

FIG. 2.-Platelet electrophoretic mobility changes induced by A.D.P. at various intervals after myocardial infarction in a man aged 64

\section{Effect of Other Inflammatory Conditions}

The parallel changes in platelet sensitivity to A.D.P. and noradrenaline which occur after myocardial infarction are apparently non-specific, for the same sequence of changes was observed in patients with pneumonia (Fig. 3), pericarditis, pulmonary embolus, venous thrombosis, and giant-cell arteritis. The length of time the abnormality persisted paralleled the clinical state of the patient: in each case the platelets were most sensitive when the patient was most ill. In the majority of cases the greatest increase in sensitivity to A.D.P. or noradrenaline was only tenfold, the maximum increase in mobility being induced by $0.005 \mu \mathrm{g} . / \mathrm{ml}$. of either agent. A thousandfold increase in sensitivity, however, occurred in a man of 35 who suffered a series of arterial and venous thromboses: at a time when there was a relatively minor superficial venous thrombosis the maximum increase in platelet mobility was induced by $0.00005 \mu \mathrm{g}$. of noradrenaline per ml., while after

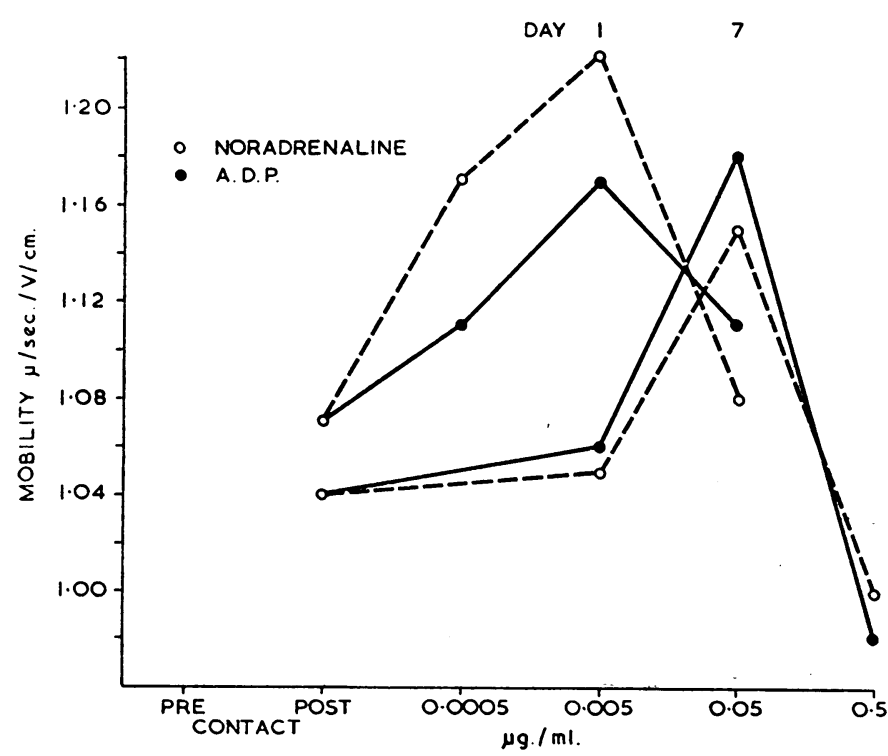

Fig. 3.-Platelet electrophoretic mobility changes induced by A.D.P. and by noradrenaline on two occasions. a week apart, in a patient with pneumonia. recovery from the illness the platelet sensitivity was normal, the maximum increase in mobility being induced by $0.05 \mu \mathrm{g}$. of noradrenaline per $\mathrm{ml}$. In all the cases there was a parallel change in sensitivity to A.D.P. and noradrenaline.

The change in platelet sensitivity to A.D.P. and noradrenaline which we observed could have been due to a change in the platelets themselves or to a change in the plasma. Since platelet-rich citrated plasma is mixed with nine volumes of some other liquid for measurement of mobility in the electrophoretic apparatus, it is simple to alter the suspending medium, and at least to dilute to a tenth any plasma factor which might be causing the change in sensitivity. We have shown (Hampton and Mitchell, 1966a) that in normal subjects the plateletrich citrated plasma can be diluted with reconstituted dry plasma, to which calcium and heparin 1 unit/ml. have been added, without affecting the mobility changes induced by A.D.P. and noradrenaline.

Blood was taken from four acutely ill patients and the platelet-rich citrated plasma was diluted in the usual way with the patient's own platelet-poor citrated plasma: in each case the maximum increase in platelet mobility was found to be induced by $0.005 \mu \mathrm{g}$. of A.D.P. or noradrenaline per $\mathrm{ml}$. Platelet-rich citrated plasma from each patient was then diluted with a reconstituted plasma mixture and the same doseresponse curves were obtained. The reverse procedure was also performed: platelet-rich citrated plasma from a normal subject was diluted with platelet-poor citrated plasma from a patient whose platelets showed an increase in sensitivity to A.D.P. The sensitivity of the platelets was found to be the same as it was when they were diluted with their own normal platelet-poor citrated plasma. It therefore appears that the abnormality observed is related to a change in the platelets, and not primarily to a change in the plasma.

\section{Effect of Surgical Operation}

A study was made of five patients (four men and one woman) who were admitted for elective surgery. Care was taken to avoid patients with active inflammatory processes, the operations involved being partial gastrectomy or vagotomy and pyloroplasty for duodenal ulcer (two cases), cholecystectomy for cholelithiasis, thyroidectomy for non-toxic nodular goitre, and haemorrhoidectomy.

To reduce the quantity of blood required for each measurement of platelet sensitivity the platelet-rich citrated plasma was diluted with reconstituted dry human plasma. This enabled more concentrations of A.D.P. and noradrenaline to be tested than were used during the study of myocardial infarction, and the concentrations of the agents causing the maximum increase in sensitivity could therefore be assessed more accurately. An additional advantage of using this substitute for platelet-poor citrated plasma was that the same mixture could be used each day, so excluding variability in the suspending inedium. The platelet response was measured the day before operation, on the first and second days after the operation, and thereafter at intervals.

The post-contact mobility of the platelets was not affected by the operations: the mean difference between successive measurements in all the cases was $3.8 \%$, which is similar to the difference observed when repeated measurements are made in normal subjects at intervals of more than 24 hours.

Pre-operatively the maximum increase of platelet mobility occurred with $0.05 \mu \mathrm{g}$. of A.D.P. and noradrenaline per $\mathrm{ml}$. in each case: the patients were therefore similar in this respect to the "normal" group which we had studied previously (Hampton and Mitchell, 1966a). The day after the operation there was a tenfold increase in sensitivity in four cases, so that the maximum increase was induced by $0.005 \mu \mathrm{g}$. of either agent per $\mathrm{ml}$. ; in the fifth case the maximum increase was induced by $0.0015 \mu \mathrm{g} . / \mathrm{ml}$. The maximum sensitivity occurred on the 
first post-operative day in all cases, and thereafter there was a progressive return to the pre-operative state. The length of time the abnormality persisted varied (Fig. 4). The two patients undergoing minor procedures (haemorrhoidectomy and thyroidectomy) were normal in four and six days respectively; the remaining three patients, in whom the surgery was more extensive, remained abnormally sensitive to A.D.P. and noradrenaline for more than six days. In all cases the maximum increase in platelet mobility was induced by the same concentration of A.D.P. and noradrenaline.

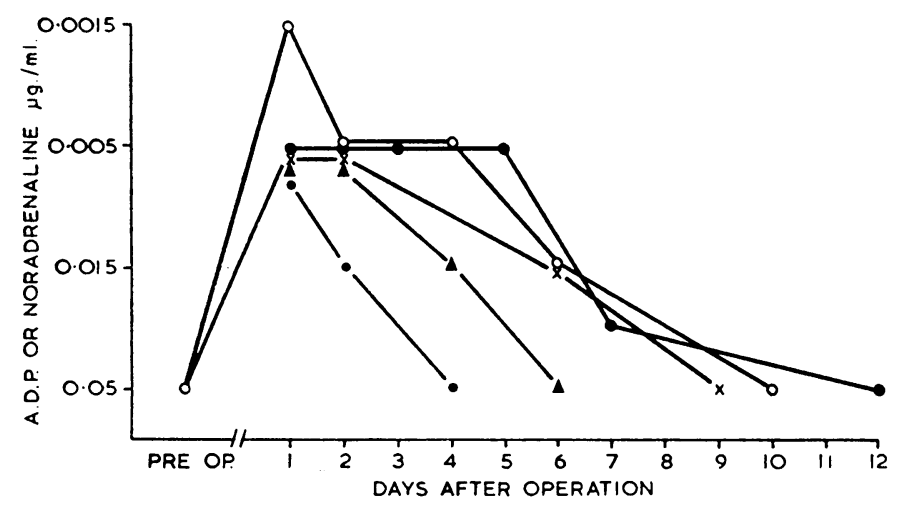

FIG. 4.-Effect of surgical operations on the concentrations of A.D.P. and of noradrenaline which induced the maximum increase in platelet mobility.

\section{Effect of Stress}

In order to determine whether mental stress might play any part in these changes, the platelet mobility responses to A.D.P. and noradrenaline were measured in three medical students three weeks before their final examinations and 30 minutes before the results were announced. There was no significant difference between the results obtained on the two occasions, and each time the maximum increase in mobility occurred with $0.05 \mu$ g. of A.D.P. and noradrenaline per $\mathrm{ml}$.

\section{Discussion}

We have suggested (Hampton and Mitchell, 1966a, 1966c) that when aggregating agents are added to platelet suspensions the resulting increase in platelet mobility, which is a measure of the increase in net negative charge, is due to the binding of negatively charged A.D.P. molecules to the platelet surface. We have shown that there appear to be two mechanisms by which this is achieved: exogenous A.D.P. is adsorbed direct, and exogenous A.T.P. is converted to A.D.P. before exerting any effect on the platelet; noradrenaline, collagen, and thrombin, on the other hand, in some way cause the release of endogenous A.D.P. on to the platelet surface.

The experiments described in this paper demonstrate that in a wide variety of pathological states the electrokinetic response of the platelet to A.D.P. and noradrenaline is in some way altered. The actual amount by which the overall net negative charge of the platelet is increased by A.D.P. and noradrenaline is little affected (Fig. 2). The number of sites at which A.D.P. molecules can be bound to the platelet is probably unaltered, and we shall present separately an estimation of the number of binding sites for A.D.P. based on similar data. In all the acute pathological states we have studied the increases in platelet sensitivity to A.D.P. and noradrenaline are precisely parallel, and it therefore seems likely that the change is related to the process which influences the binding of either exogenous or endogenous A.D.P. The changes induced by A.D.P. and noradrenaline in normal subjects have a characteristic time course, mobility returning to normal in 30 minutes. The net sensitivity to the agents at any instant must therefore reflect the algebraic sum of adsorption of A.D.P. to the platelet surface and removal of A.D.P. from the surface, either by degrada- tion or by its entry into the platelet. The increased sensitivity which we have observed might indicate that A.D.P. is more readily adsorbed, a lower concentration than in normal patients sufficing to place it on the platelet surface. Alternatively, the sensitivity may be increased because removal of adsorbed A.D.P. may be slower in the abnormal patients. As the changes observed with A.D.P. are precisely paralleled by those found with noradrenaline, the increased avidity of the platelet for exogenous A.D.P. or its failure to remove the exogenous agent must also apply to the endogenous A.D.P. released by noradrenaline.

The means by which this change is brought about are not clear. The increase in sensitivity occurs within 12 hours of the onset of the inflammatory state, and it is therefore unlikely to be due to the appearance of a new generation of platelets. The time of onset of the change may parallel the rise in fibrinogen which occurs in inflammatory states (Meyers, 1948), but the fibrinogen level often remains high for considerably longer than the 10 days for which we have found the platelets to remain abnormal. The increased sensitivity of platelets in myocardial infarction occurs sooner than the rise in erythrocyte sedimentation rate or the rise in plasma transaminase. The time course of the change in platelet sensitivity after surgical operations is also quite different from the change in platelet adhesiveness and aggregation observed by Payling Wright (1942) and Emmons and Mitchell (1965), where the maximum change occurred after 10 days (the time when the platelet count is highest and a new generation of platelets may be being studied). The course of the platelet-sensitivity changes shows some similarity to the increased fibrinolytic activity which occurs in a wide variety of conditions (Fearnley, 1965), but if platelet sensitivity were related to fibrinolysis some change would have been expected in the "stressed" medical students.

It must be concluded that the electrophoretic method of investigating platelet behaviour measures something not amenable to measurement by the other systems commonly in use for studying platelets. Further clinical studies with the electrophoretic apparatus are in progress to determine the nature and significance of these mobility changes.

\section{Summary}

The effect of A.D.P. and noradrenaline on the electrokinetic response of human platelets has been studied in patients suffering from a variety of acute illnesses. In inflammatory states such as pneumonia and after myocardial infarction and surgical operations there is a considerable (up to a hundredfold) increase in the sensitivity of platelets to these agents, as estimated by the concentration required to produce a maximum increase in mobility. This increase in sensitivity is maximal 12 hours from the onset of the inflammatory state, and in the case of surgical procedures the platelets have returned to their preoperative state within 12 days. Possible mechanisms involved in this change in sensitivity are discussed.

We are grateful to the physicians and surgeons of the Radcliffe Infirmary, Oxford, for allowing us to study patients under their care, and to the medical students who were studied during a period of stress.

\section{REFERENCES}

Bangham, A. D., Flemans, R., Heard, D. H., and Seaman, G. V. F. (1958). Nature (Lond.), 182, 642.

Emmons, P. R., and Mitchell, J. R. A. (1965). Lancet, 1, 71.

Fearnley, G. R. (1965). Fibrinolysis. Arnold, London.

Field, E. J., and Caspary, E. A. (1964). Lancet, 2, 876.

Hampton, J. R., and Mitchell, J. R. A. (1966a). Brit. med. F., 1, 1074. Hame (1966b). Nature (Lond.), 209, 470.

- (1966c). Ibid. In press.

Hirsh, J., and McBride, J. A. (1965). Brit. med. F., 2, 797.

McDonald, L., Bray, C., Field, C., Love, F., and Davies, B. (1964). Lancet, 1, 745 .

and Edgill, M. (1957). Ibid., 2, 457.

Meyers, L. (1948). Arch. intern. Med., 82, 419.
Wright, H. P. (1942). f. Path. Bact., 54, 461. 